\title{
A New Generalized Modified Weibull Distribution
}

\author{
Morad Alizadeh ${ }^{1, *}$, Muhammad Nauman Khan ${ }^{2}$, Mahdi Rasekhi ${ }^{3}$, G.G Hamedani ${ }^{4}$ \\ ${ }^{1}$ Department of Statistics, Persian Gulf University, Bushehr, 75169, Iran \\ ${ }^{2}$ Department of Mathematics, Kohat University of Science \& Technology, Kohat, Pakistan \\ ${ }^{3}$ Department of Statistics, Faculty of Mathematical Sciences and Statistics, Malayer University, Malayer, Iran \\ ${ }^{4}$ Department of Mathematics, Statistics and Computer Science, Marquette University, USA
}

\begin{abstract}
We introduce a new distribution, so called A new generalized modified Weibull (NGMW) distribution. Various structural properties of the distribution are obtained in terms of Meijer's $G$-function, such as moments, moment generating function, conditional moments, mean deviations, order statistics and maximum likelihood estimators. The distribution exhibits a wide range of shapes with varying skewness and assumes all possible forms of hazard rate function. The NGMW distribution along with other distributions are fitted to two sets of data, arising in hydrology and in reliability. It is shown that the proposed distribution has a superior performance among the compared distributions as evidenced via goodness-of-fit tests.
\end{abstract}

Keywords Odd log-logistic distribution, modified Weibull distribution, moment, simulation study, maximum likelihood estimation

AMS 2010 subject classifications 60E05, 62E15

DOI: $10.19139 /$ soic-2310-5070-1014

\section{Introduction}

The Weibull distribution is a popular distribution for modeling and analyzing lifetime data with monotonic hazard rates. On the other hand, for complex systems, the hazard rate function can often be of non-monotonic shape, which the Weibull distribution cannot accommodate. To overcome such shortcomings, various generalizations of the classical Weibull distribution have been investigated by several authors in the recent years; among them, the extended flexible Weibull distribution [2], the generalized modified Weibull distribution [3], the exponentiated Weibull distribution [17], the additive Weibull distribution [20], Weibull Birnbaum-Saunders distribution [12] and the modified beta Weibull distribution [11] and new type 1 half-logistic weibull [1]. Also, Sarhan and Zaindin [28] introduced the modified-Weibull (MW) distribution having three parameters $\beta>0, \lambda>0$ and $\gamma>0$, with the cumulative distribution function (cdf) and probability density function (pdf)

$$
G(x)=1-\mathrm{e}^{-\beta x-\lambda x^{\gamma}}
$$

and

$$
g(x)=\left(\beta+\lambda \gamma x^{\gamma-1}\right) \mathrm{e}^{-\beta x-\lambda x^{\gamma}}, x>0,
$$

respectively. It is worth noting that the MW distribution has submodels the exponential $(\lambda=0)$ and Weibull $(\beta=0)$.

\footnotetext{
${ }^{*}$ Correspondence to: Morad Alizadeh(Email: m.alizadeh@pgu.ac.ir ). Department of Statistics, Persian Gulf University, Bushehr, 75169, Iran.
}

ISSN 2310-5070 (online) ISSN 2311-004X (print)

Copyright (C) 2021 International Academic Press 
Consider starting form a parent continuous cdf $\mathrm{G}(\mathrm{x})$, where $\bar{G}(x)=1-G(x)$ and $\alpha>0$. The cdf of generalized odd log-logistic family of distributions (Gleaton and Lynch [10]) is defined by

$$
F(x)=\frac{G(x)^{\alpha}}{G(x)^{\alpha}+\theta \bar{G}(x)^{\alpha}} .
$$

Its corresponding pdf is

$$
f(x)=\frac{\alpha \theta g(x) G(x)^{\alpha-1} \bar{G}(x)^{\alpha-1}}{\left\{G(x)^{\alpha}+\theta \bar{G}(x)^{\alpha}\right\}^{2}},
$$

where $g(x)=\mathrm{d} G(x) / \mathrm{d} x$ is the density of the baseline distribution.

Here, we introduce a new distribution having four parameters, so called A new generalized modified Weibull (NGMW) distribution, which is a generalization for the MW distribution. Our motivation for introducing the NGMW distribution is due to the simple analytic expressions of $G(x)$ and $g(x)$ of the MW distribution and it can be represented as infinite linear combination of MW distributions. Furthermore, the distribution provides a wide range of shapes with varying skewness, varied tail weights and shifting modes based on its additional parameters. It also accommodates most forms of hazard rates that are encountered in a variety of real-life problems.

The NGMW distribution is obtained by taking $G(x)$ in (3) to be the cdf (1) of the MW distribution. Accordingly, the cdf of the NGMW distribution is

$$
F(x)=\frac{\left(1-\mathrm{e}^{-\beta x-\lambda x^{\gamma}}\right)^{\alpha}}{\left(1-\mathrm{e}^{-\beta x-\lambda x^{\gamma}}\right)^{\alpha}+\theta \mathrm{e}^{-\alpha \beta x-\alpha \lambda x^{\gamma}}} .
$$

The NGMW density function is

$$
f(x)=\frac{\alpha \theta\left(\beta+\gamma \lambda x^{\gamma-1}\right) \mathrm{e}^{-\alpha \beta x-\alpha \lambda x^{\gamma}}\left(1-\mathrm{e}^{-\beta x-\lambda x^{\gamma}}\right)^{\alpha-1}}{\left\{\left(1-\mathrm{e}^{-\beta x-\lambda x^{\gamma}}\right)^{\alpha}+\theta \mathrm{e}^{-\alpha \beta x-\alpha \lambda x^{\gamma}}\right\}^{2}}, x>0 .
$$

The survival function and the hazard rate function of the NGMW distribution are

$$
S(x)=\frac{\theta \mathrm{e}^{-\alpha \beta x-\alpha \lambda x^{\gamma}}}{\left(1-\mathrm{e}^{-\beta x-\lambda x^{\gamma}}\right)^{\alpha}+\mathrm{e}^{-\alpha \beta x-\alpha \lambda x^{\gamma}}}
$$

and

$$
\tau(x)=\frac{\alpha \theta\left(\beta+\gamma \lambda x^{\gamma-1}\right)\left(1-\mathrm{e}^{-\beta x-\lambda x^{\gamma}}\right)^{\alpha-1}}{\left(1-\mathrm{e}^{-\beta x-\lambda x^{\gamma}}\right)^{\alpha}+\theta \mathrm{e}^{-\alpha \beta x-\alpha \lambda x^{\gamma}} .} .
$$

An interpretation of the NGMW family (5) can be given as follows. Let $T$ be a random variable describing a stochastic system with cdf $G(x)=1-\mathrm{e}^{-\beta \mathrm{x}-\lambda \mathrm{x}^{\gamma}}$. If the random variable $X$ represents the odds ratio, the risk that the system following the lifetime $T$ will not working at time $x$ is given by $G(x) /[1-G(x)]$. If we are interested in modeling the randomness of the odds ratio by the Marshall-Olkin log-logistic pdf $r(t)=\alpha \theta t^{\alpha-1} /\left(1+\theta t^{\alpha}\right)^{2}$ (and cdf $R(t)=\frac{t^{\alpha}}{1+\theta t^{\alpha}}$, for $t>0$ ), the cdf of $X$ is given by

$$
\operatorname{Pr}(X \leq x)=R\left(\frac{G(x)}{1-G(x)}\right),
$$

which is exactly the cdf (5) of the new family.

The remainder of this article is organized as follows. Some statistical functions of the NGMW distribution are provided in Section 2, such as the quantile function, moments, moment generating function, conditional moments, mean deviations, reliability curves and the distribution of order statistics. Certain characterizations of the proposed distribution are presented in section 3. The estimation of the parameters by maximum likelihood (ML) method is investigated in Section 4. Simulation study is performed in Section 5. In Section 6, the NGMW distribution along with other distributions are fitted to two sets of data. It is shown that, the proposed distribution has a better fit as verified by several goodness-of-fit tests. 
Table 1. Some special cases of the NGMW distribution

\begin{tabular}{|c|c|c|c|c|c|}
\hline$\alpha$ & $\beta$ & $\lambda$ & $\gamma$ & $\theta$ & Reduced distribution \\
\hline 1 & - & 0 & - & 1 & Exponential distribution \\
\hline 1 & - & 0 & - & - & Marshall-Olkin Exponential distribution \\
\hline 1 & 0 & - & - & 1 & Weibull distribution \\
\hline 1 & 0 & - & - & - & Marshall-Olkin Weibull distribution \\
\hline 1 & 0 & - & 2 & 1 & Rayleigh distribution \\
\hline 1 & 0 & - & 2 & - & Marshall-Olkin Rayleigh distribution \\
\hline 1 & - & - & 2 & 1 & Linear failure rate distribution \\
\hline 1 & - & - & 2 & - & Marshall-Olkin Linear failure rate distribution \\
\hline 1 & - & - & - & 1 & Exponential weibull distribution \\
\hline 1 & - & - & - & - & Marshall-Olkin Exponential weibull distribution \\
\hline- & - & 0 & - & 1 & OLL exponential distribution [9] \\
\hline- & - & 0 & - & - & Marshall-Olkin OLL exponential distribution [10] \\
\hline- & 0 & - & - & 1 & OLL weibull distribution [9] \\
\hline- & 0 & - & - & - & Marshall-Olkin OLL weibull distribution [10] \\
\hline- & 0 & - & 2 & 1 & OLL Rayleigh distribution [9] \\
\hline- & 0 & - & 2 & - & Marshall-Olkin OLL Rayleigh distribution [10] \\
\hline- & - & - & 2 & 1 & OLL linear failure rate [9] \\
\hline- & - & - & 2 & - & Marshall-Olkin OLL linear failure rate [10] \\
\hline 1 & - & - & - & - & Marshall-Olkin MW [25] \\
\hline- & - & - & - & 1 & OLLMW [26] \\
\hline
\end{tabular}

\section{Structural properties of the NGMW distribution}

Using generalized binomial expansion we can write

$$
\left(1-\mathrm{e}^{-\beta x-\lambda x^{\gamma}}\right)^{\alpha}=\sum_{j=0}^{\infty} a_{j}\left(1-\mathrm{e}^{-\beta x-\lambda x^{\gamma}}\right)^{j},
$$

where $a_{j}=\sum_{i=j}^{\infty}(-1)^{i+j}\left(\begin{array}{c}\alpha \\ i\end{array}\right)\left(\begin{array}{l}i \\ j\end{array}\right)$ and

$$
\left(1-\mathrm{e}^{-\beta x-\lambda x^{\gamma}}\right)^{\alpha}+\theta \mathrm{e}^{-\alpha \beta x-\alpha \lambda x^{\gamma}}=\sum_{j=0}^{\infty} b_{j}\left(1-\mathrm{e}^{-\beta x-\lambda x^{\gamma}}\right)^{j},
$$

where $b_{j}=a_{j}+\theta(-1)^{j}\left(\begin{array}{c}\alpha \\ j\end{array}\right)$. Therefore, we can write

$$
\begin{aligned}
F(x) & =\frac{\sum_{j=0}^{\infty} a_{j}\left[1-\mathrm{e}^{-\beta x-\lambda x^{\gamma}}\right]^{j}}{\sum_{j=0}^{\infty} b_{j}\left[1-\mathrm{e}^{-\beta x-\lambda x^{\gamma}}\right]^{j}}=\sum_{j=0}^{\infty} c_{j}\left[1-\mathrm{e}^{-\beta x-\lambda x^{\gamma}}\right]^{j} \\
& =\sum_{j=0}^{\infty} c_{j} H_{j}(x)
\end{aligned}
$$

where $c_{0}=\frac{a_{0}}{b_{0}}$ and for $j \geq 1$,

$$
c_{j}=b_{0}^{-1}\left(a_{j}-b_{0}^{-1} \sum_{r=1}^{j} b_{r} c_{j-r}\right)
$$

and

$$
H_{j}(x)=\left(1-\mathrm{e}^{-\beta x-\lambda x^{\gamma}}\right)^{j}
$$



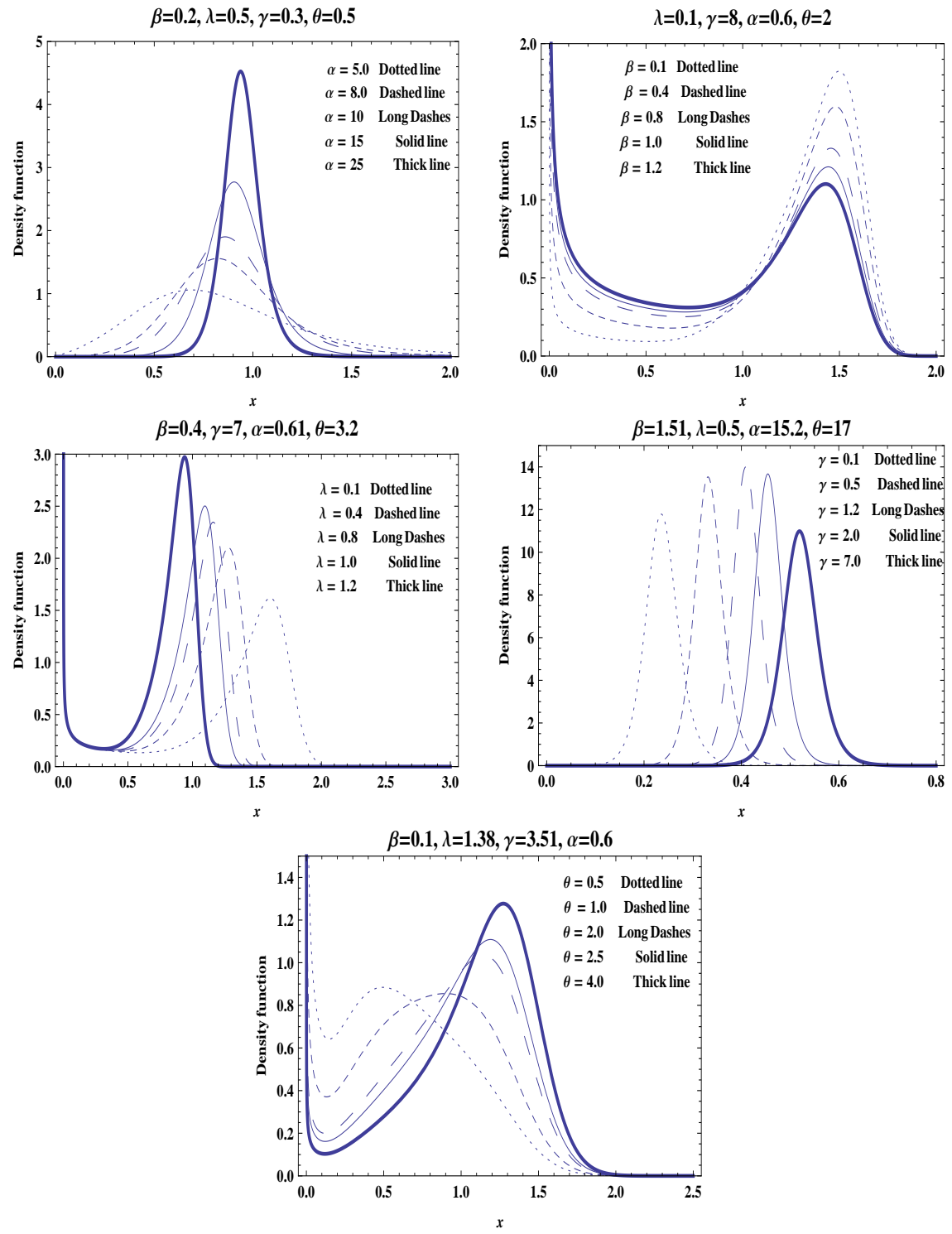

Figure 1. Plots of the NGMW pdf for some parameter values

The pdf specified by (6) can be written as

$$
f(x)=\sum_{j=0}^{\infty} c_{j+1} h_{j+1}(x)
$$

and $h_{j+1}(x)=(j+1)\left(\beta+\lambda \gamma x^{\gamma-1}\right) \mathrm{e}^{-\beta x-\lambda x^{\gamma}}\left(1-\mathrm{e}^{-\beta x-\lambda x^{\gamma}}\right)^{j}$.

It is clear from (11) that $f(x)$ can be expressed as infinite linear combinations of exponentiated MW distributions and hence many properties of the NGMW can be deduced from the corresponding ones of the MW distribution. In what follows, we discuss some properties of the NGMW distribution and consider several associated statistical functions. 

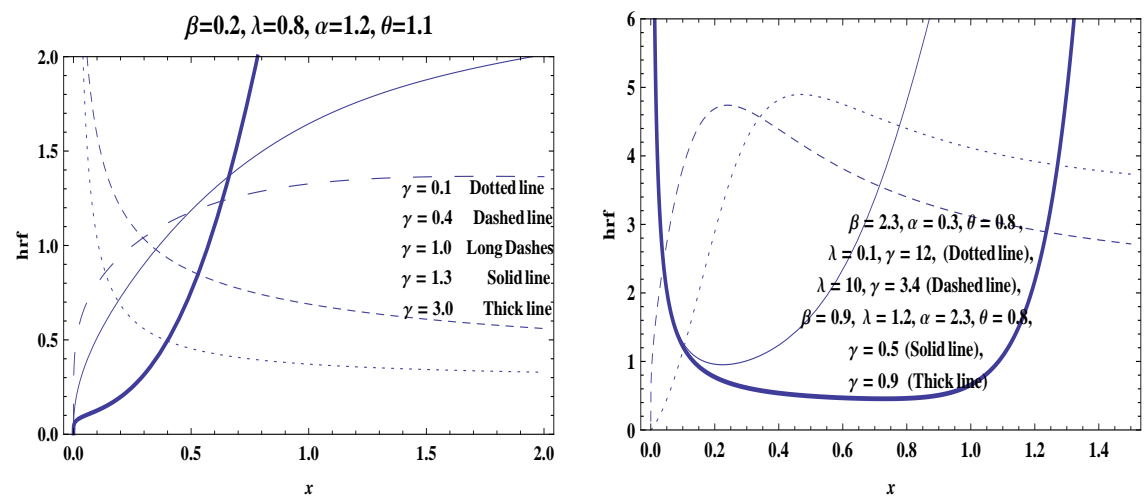

Figure 2. Plots of the NGMW hrf for some parameter values

If $X \sim$ NGMW, then one can easily show that the random variable $Y=\left(\mathrm{e}^{\beta \mathrm{x}+\lambda \mathrm{x}^{\gamma}}-1\right)$ has Marshall-Olkin $\log$-logistic distribution with shape parameter $\alpha$ and scale parameter equal 1 .

\subsection{Asymptotic and Shapes}

In this section, we discuss the asymptotic and possible shapes of the pdf (6) and the hazard rate function (8).

The asymptotics of equations (5), (6) and (8) as $x \rightarrow 0$ are given by

$$
\begin{array}{ll}
F(x) \sim \frac{\left(\beta x+\lambda x^{\gamma}\right)^{\alpha}}{\theta} & \text { as } \quad \mathrm{x} \rightarrow 0, \\
f(x) \sim \frac{\alpha\left(\beta+\lambda \gamma x^{\gamma-1}\right)\left(\beta x+\lambda x^{\gamma}\right)^{\alpha-1}}{\theta} & \text { as } \quad \mathrm{x} \rightarrow 0, \\
h(x) \sim \frac{\alpha\left(\beta+\lambda \gamma x^{\gamma-1}\right)\left(\beta x+\lambda x^{\gamma}\right)^{\alpha-1}}{\theta} & \text { as } \quad \mathrm{x} \rightarrow 0,
\end{array}
$$

The asymptotics of equations (5), (6) and (8) as $x \rightarrow \infty$ are given by

$$
\begin{array}{ll}
1-F(x) \sim \theta \mathrm{e}^{-\alpha\left(\beta \mathrm{x}+\lambda \mathrm{x}^{\gamma}\right)} & \text { as } \quad \mathrm{x} \rightarrow \infty, \\
f(x) \sim \alpha \theta\left(\beta+\lambda \gamma x^{\gamma-1}\right) \mathrm{e}^{-\alpha\left(\beta \mathrm{x}+\lambda \mathrm{x}^{\gamma}\right)} & \text { as } \quad \mathrm{x} \rightarrow \infty, \\
h(x) \sim \alpha \theta\left(\beta+\lambda \gamma x^{\gamma-1}\right) & \text { as } \quad \mathrm{x} \rightarrow \infty .
\end{array}
$$

Observed that plots (a), (b), (c) and (d) indicate how the parameters $\beta, \lambda, \gamma$ and $\alpha$ affect the NGMW density and show flexibility of density shapes where skewness, heavy tails and modality can be observed. Figures (e) and (f) represent increasing, decreasing, unimodal, v-shaped, upside-down bathtub and bathtub-shaped hazard rate function.

\subsection{Quantile function}

For simulation of NGMW, if $U \sim u(0,1)$, then the solution of non-linear equation

$$
\beta x+\lambda x^{\gamma}+\log \left[\frac{(1-u)^{\frac{1}{\alpha}}}{(\theta u)^{\frac{1}{\alpha}}+(1-u)^{\frac{1}{\alpha}}}\right]=0
$$

has $\operatorname{cdf}(5)$. 


\subsection{Moments and moment generating function}

We now obtain representations of the moments and moment generating function of the NGMW random variable on the basis of the result developed in [19]:

$$
\begin{aligned}
\int_{0}^{\infty} x^{\eta-1} \mathrm{e}^{-\theta x^{k}} \mathrm{e}^{s x} \mathrm{dx}= & \frac{(2 \pi)^{1-(\mathrm{q}+\mathrm{p}) / 2} \mathrm{q}^{1 / 2} \mathrm{p}^{\eta-1 / 2}}{(-\mathrm{s})^{\eta}} \\
& \times G_{p, q}^{q, p}\left(\left(-\frac{p}{s}\right)^{p}\left(\frac{\theta}{q}\right)^{q} \mid \begin{array}{cc}
1-\frac{i+\eta}{p}, & i=0,1, \ldots, p-1 \\
j / q, & j=0,1, \ldots, q-1
\end{array}\right),
\end{aligned}
$$

where $\Re(\eta), \Re(\theta), \Re(s)<0$ and $k=\frac{p}{q}$ is a rational number with $p$ and $q \neq 0$ integers.

Making use of (13), the $r^{\text {th }}$ order moment and moment generating function of the NGMW distribution can be expressed in terms of Meijer's $G$-functions as

$$
\begin{aligned}
E\left(X^{r}\right) & =\beta \sum_{j=0}^{\infty} \sum_{k=0}^{j}(-1)^{k}(j+1) c_{j+1}\left(\begin{array}{l}
j \\
k
\end{array}\right) \frac{(2 \pi)^{1-(q+p) / 2} q^{1 / 2} p^{r+1 / 2}}{(\beta(k+1))^{r+1}} \\
& \times G_{p, q}^{q, p}\left(\left(\frac{p}{\beta(k+1)}\right)^{p}\left(\frac{\lambda(k+1)}{q}\right)^{q} \mid \begin{array}{cc}
1-\frac{i+r+1}{p}, & i=0,1, \ldots, p-1 \\
j / q, & j=0,1, \ldots, q-1
\end{array}\right) \\
& +\lambda \gamma \sum_{j=0}^{\infty} \sum_{k=0}^{j}(-1)^{k}(j+1) c_{j+1}\left(\begin{array}{l}
j \\
k
\end{array}\right) \frac{(2 \pi)^{1-(q+p) / 2} q^{1 / 2} p^{r+\gamma-1 / 2}}{(\beta(k+1))^{r+\gamma}} \\
& \times G_{p, q}^{q, p}\left(\left(\frac{p}{\beta(k+1)}\right)^{p}\left(\frac{\lambda(k+1)}{q}\right)^{q} \mid \begin{array}{cc}
1-\frac{i+r+\gamma}{p}, & i=0,1, \ldots, p-1 \\
j / q, & j=0,1, \ldots, q-1
\end{array}\right),
\end{aligned}
$$

The $h^{t h}$ order moment can readily be determined by replacing $r$ with $-h$ in (14). The moment generating function of the NGMW is

$$
\begin{aligned}
M(t) & =\beta \sum_{j=0}^{\infty} \sum_{k=0}^{j}(-1)^{k}(j+1) c_{j+1}\left(\begin{array}{l}
j \\
k
\end{array}\right) \frac{(2 \pi)^{1-(q+p) / 2} q^{1 / 2} p^{1 / 2}}{\beta(k+1)-t} \\
& \times G_{p, q}^{q, p}\left(\left(\frac{p}{\beta(k+1)-t}\right)^{p}\left(\frac{\lambda(k+1)}{q}\right)^{q} \mid \begin{array}{cc}
1-\frac{i+1}{p}, & i=0,1, \ldots, p-1 \\
j / q, & j=0,1, \ldots, q-1
\end{array}\right) \\
& +\lambda \gamma \sum_{j=0}^{\infty} \sum_{k=0}^{j}(-1)^{k}(j+1) c_{j+1}\left(\begin{array}{l}
j \\
k
\end{array}\right) \frac{(2 \pi)^{1-(q+p) / 2} q^{1 / 2} p^{\gamma-1 / 2}}{((n+\rho) \lambda-t)^{\gamma}} \\
& \times G_{p, q}^{q, p}\left(\left(\frac{p}{\beta(k+1)-t}\right)^{p}\left(\frac{\lambda(k+1)}{q}\right)^{q} \mid \begin{array}{cc}
1-\frac{i+\gamma}{p}, & i=0,1, \ldots, p-1 \\
j / q, & j=0,1, \ldots, q-1
\end{array}\right)
\end{aligned}
$$




\subsection{Order statistics}

Suppose $X_{1}, \ldots, X_{n}$ is a random sample from the NGMW family of distributions. Denote the ordered random variables in the ascending as $X_{1: n} \leq, \ldots, \leq X_{n: n}$; the pdf of $X_{i: n}$ is (David and Nagarajah [6])

$$
\begin{aligned}
f_{i: n}(x) & =K f(x) F^{i-1}(x)\{1-F(x)\}^{n-i}=K \sum_{j=0}^{n-i}(-1)^{j}\left(\begin{array}{c}
n-i \\
j
\end{array}\right) f(x) F(x)^{j+i-1} \\
& =\sum_{r, k=0}^{\infty} \sum_{j=0}^{n-i} m_{j, r, k} h_{r+k+1}(x)
\end{aligned}
$$

where $K=n ! /[(i-1) !(n-i) !]$.

Here $h_{r+k+1}(x)$ denotes the exp-MW density function with power parameter $r+k+1$,

$$
m_{j, r, k}=\frac{(-1)^{j} n !}{(i-1) !(n-i-j) ! j !} \frac{(r+1) c_{r+1} f_{j+i-1, k}}{[r+k+1]},
$$

and $c_{k}$ is defined by Equation (10). Here, the quantities $f_{j+i-1, k}$ is obtained recursively by $f_{j+i-1,0}=c_{0}^{j+i-1}$ and (for $k \geq 1$ )

$$
f_{j+i-1, k}=\left(k c_{0}\right)^{-1} \sum_{m=1}^{k}[m(j+i)-k] c_{m} f_{j+i-1, k-m} .
$$

Thus one can easily obtain moments, generating function and incomplete moment of order statistics of NGMW. In the remainder of this section, we shall make use of the next lemma.

\section{Lemma 1}

Let

$$
\begin{aligned}
J(x ; r, \theta) & =\int_{0}^{x} y^{r} f(y) \mathrm{d} y=\sum_{j, l=0}^{\infty} \sum_{k=0}^{j}(j+1) c_{j+1}\left(\begin{array}{l}
j \\
k
\end{array}\right) \frac{(-1)^{k+l}}{l !}\{\beta(k+1)\}^{l} \\
& \times \int_{0}^{x} y^{l+r}\left(\beta+\lambda \gamma y^{\gamma-1}\right) \mathrm{e}^{-\lambda(k+1) y^{\gamma}} \mathrm{d} y, \quad r=1,2, \ldots,
\end{aligned}
$$

where $\theta:=(\beta, \gamma, \lambda, \alpha)$. Then, we have

$$
\begin{aligned}
J(x ; r, \theta) & =\sum_{j, l=0}^{\infty} \sum_{k=0}^{j}(j+1) c_{j+1}\left(\begin{array}{l}
j \\
k
\end{array}\right) \frac{(-1)^{k+l}}{l !}\{\beta(k+1)\}^{l} \\
& \times\left\{\frac{\beta q x^{p(l+r+1)}}{p(2 \pi)^{(q-1) / 2}}\right. \\
& \times G_{p, p+q}^{q, p}\left(\frac{(\lambda(k+1))^{q} x^{p}}{q^{q}} \mid \begin{array}{l|l}
\frac{-l-r}{p}, \frac{1-l-r}{p}, \ldots, \frac{p-l-r-1}{p},- \\
0, \frac{-l-r-1}{p}, \frac{l+r}{p}, \ldots, \frac{p-l-r-2}{p}
\end{array}\right) \\
& +\frac{\lambda x^{p(l+r+\gamma)}}{(2 \pi)^{(q-1) / 2}} \\
& \left.\times G_{p, p+q}^{q, p}\left(\frac{(\lambda(k+1))^{q} x^{p}}{q^{q}} \mid \begin{array}{l|l}
\frac{-l-r-\gamma+1}{p}, \frac{2-l-r-\gamma}{p}, \ldots, \frac{p-l-r-\gamma}{p},- \\
p
\end{array}\right)\right\}
\end{aligned}
$$


Proof

The proof follows ny considering the arbitrary function $\mathrm{e}^{-g(x)}=G_{0,1}^{1,0}\left(g(x) \mid \begin{array}{c}- \\ 0\end{array}\right)$. letting $k=p / q$ where $p \geq 1, q \geq 1$ are natural co-prime numbers and making use of the identity

$$
\begin{aligned}
& \int_{0}^{x} y^{t} G_{0,1}^{1,0}\left(\lambda(k+1) y^{p / q} \mid \begin{array}{c}
- \\
0
\end{array}\right) \mathrm{d} y \\
& =\frac{q x^{p(t+1)}}{p(2 \pi)^{(q-1) / 2}} G_{p, p+q}^{q, p}\left(\begin{array}{l|l}
\frac{(\lambda(k+1))^{q} x^{p}}{q^{q}} & \left.\begin{array}{l}
\frac{-t}{p}, \frac{1-t}{p}, \ldots, \frac{p-t-1}{p},- \\
0, \frac{-t-1}{p}, \frac{t}{p}, \ldots, \frac{p-t-2}{p}
\end{array}\right),
\end{array}\right.
\end{aligned}
$$

which results from Equation (13) of [4].

\subsection{Conditional moments and mean deviations}

In connection with lifetime distributions, it is important to determine the conditional moments $E\left(X^{r} \mid X>t\right)$, $r=1,2, \cdots$, which are of interest in predictive inference. The $r^{t h}$ conditional moment of the NGMW distribution can be obtained as

$$
\begin{aligned}
E\left(X^{r} \mid X>t\right) & =\frac{1}{S(t)}\left[E\left(X^{r}\right)-\int_{0}^{t} x^{r} f(x) \mathrm{d} x\right] \\
& =\frac{\left(1-\mathrm{e}^{-\beta x-\lambda x^{\gamma}}\right)^{\alpha}+\mathrm{e}^{-\alpha \beta x-\alpha \lambda x^{\gamma}}}{\mathrm{e}^{-\alpha \beta x-\alpha \lambda x^{\gamma}}}\left[E\left(X^{r}\right)-J(t ; r, \theta)\right] .
\end{aligned}
$$

The mean deviations provide useful information about the characteristics of a population and it can be calculated from the first incomplete moment. Indeed, the amount of dispersion in a population may be measured to some extent by all the deviations from the mean and median. The mean deviations of $X$ about the mean $\mu=E(X)$ and about the median $M$ can be expressed as $\delta=2 \mu F(\mu)-2 m(\mu)$ and $\eta=\mu-2 m(M)$, where $F(\mu)$ is calculated from (5) and

$$
m(z)=\int_{0}^{z} x f(x) d x=J(z ; 1, \theta) .
$$

\subsection{Reliability curves}

The Bonferroni and Lorenz curves have various applications in economics, reliability, insurance and medicine.

The Bonferroni curve $B_{F}[F(x)]$ for the NGMW distribution is given by

$$
B_{F}[F(x)]=\frac{1}{E(X) \cdot F(x)} \int_{0}^{x} y f(y) \mathrm{d} y=\frac{J(x ; 1, \theta)}{E(X) \cdot F(x)},
$$

and the Lorenz curve of $F$ is

$$
L_{F}[F(x)]=\frac{J(x ; 1, \theta)}{E(X)} .
$$

The scaled total time on test transform of a distribution function $F$ [?] is defined by $S_{F}[F(t)]=\frac{1}{E(X)} \int_{0}^{t} \bar{F}(y) d y$, and it is important for the ageing properties of the underlying distribution and can be applied to solve geometrically some stochastic maintenance problems.

\section{Characterizations of NGMW Distribution}

This section deals with various characterizations of NGMW distribution. These characterizations are based on: $(i)$ a simple relationship between two truncated moments; (ii) the hazard function and (iii) the reverse (or reversed) hazard function. It should be mentioned that for characterization $(i)$ the cdf may not have a closed form.

We present our characterizations $(i)-(i i i)$ in three subsections. 


\subsection{Characterizations based on two truncated moments}

In this subsection we present characterizations of NGMW distribution in terms of the ratio of two truncated moments. This characterization result employs a theorem due to Glänzel [7], see Theorem 1 in Appendix B. Note that the result holds also when the interval $H$ is not closed. As shown in [7], this characterization is stable in the sense of weak convergence.

proposition 1

Let $\quad X: \Omega \rightarrow(0, \infty)$ be a continuous random variable and let, $h(x)=$ $\left(\mathrm{e}^{-\beta x-\lambda x^{\gamma}}\right)^{1-\alpha}\left\{\left(1-\mathrm{e}^{-\beta x-\lambda x^{\gamma}}\right)^{\alpha}+\theta \mathrm{e}^{-\alpha \beta x-\alpha \lambda x^{\gamma}}\right\}^{2}$ and $g(x)=h(x)\left(1-\mathrm{e}^{-\beta x-\lambda x^{\gamma}}\right)^{\alpha}$ for $x>0$. The random variable $X$ has pdf (6) if and only if the function $\xi$ defined in Theorem 1 has the form

$$
\xi(x)=\frac{1}{2}\left\{1+\left(1-\mathrm{e}^{-\beta x-\lambda x^{\gamma}}\right)^{\alpha}\right\}, \quad x>0 .
$$

Proof

If $X$ has pdf (6), then

$$
(1-F(x)) E[h(x) \mid X \geq x]=\theta\left\{1-\left(1-\mathrm{e}^{-\beta x-\lambda x^{\gamma}}\right)^{\alpha}\right\}, \quad x>0,
$$

and

and finally

$$
(1-F(x)) E[g(x) \mid X \geq x]=\frac{\theta}{2}\left\{1-\left(1-\mathrm{e}^{-\beta x-\lambda x^{\gamma}}\right)^{2 \alpha}\right\}, \quad x>0
$$

$$
\xi(x) h(x)-g(x)=\frac{1}{2} h(x)\left\{1-\left(1-\mathrm{e}^{-\beta x-\lambda x^{\gamma}}\right)^{\alpha}\right\}>0 \text { for } x>0 .
$$

Conversely, if $\xi$ is given as above, then

$$
s^{\prime}(x)=\frac{\xi^{\prime}(x) h(x)}{\xi(x) h(x)-g(x)}=\frac{\alpha\left(\beta+\gamma \lambda x^{\gamma-1}\right) \mathrm{e}^{-\beta x-\lambda x^{\gamma}}\left(1-\mathrm{e}^{-\beta x-\lambda x^{\gamma}}\right)^{\alpha-1}}{1-\left(1-\mathrm{e}^{-\beta x-\lambda x^{\gamma}}\right)^{\alpha}} \quad x>0,
$$

and hence

$$
s(x)=-\log \left\{1-\left(1-\mathrm{e}^{-\beta x-\lambda x^{\gamma}}\right)^{\alpha}\right\}, \quad x>0 .
$$

Now, in view of Theorem 1, $X$ has density (6).

\section{Corollary 1}

Let $X: \Omega \rightarrow(0, \infty)$ be a continuous random variable and let $h(x)$ be as in Proposition 1 . Then, $X$ has pdf 6 if and only if there exist functions $g$ and $\xi$ defined in Theorem 1 satisfying the differential equation

$$
\frac{\xi^{\prime}(x) h(x)}{\xi(x) h(x)-g(x)}=\frac{\alpha\left(\beta+\gamma \lambda x^{\gamma-1}\right) \mathrm{e}^{-\beta x-\lambda x^{\gamma}}\left(1-\mathrm{e}^{-\beta x-\lambda x^{\gamma}}\right)^{\alpha-1}}{1-\left(1-\mathrm{e}^{-\beta x-\lambda x^{\gamma}}\right)^{\alpha}}, \quad x>0 .
$$

Proof

The general solution of the differential equation in Corollary 1 is

$$
\begin{aligned}
\xi(x) & =\left\{1-\left(1-\mathrm{e}^{-\beta x-\lambda x^{\gamma}}\right)^{\alpha}\right\}^{-1} \\
& \times\left[-\int \alpha\left(\beta+\gamma \lambda x^{\gamma-1}\right) \mathrm{e}^{-\beta x-\lambda x^{\gamma}}\left(1-\mathrm{e}^{-\beta x-\lambda x^{\gamma}}\right)^{\alpha-1}(h(x))^{-1} g(x)+D\right],
\end{aligned}
$$

where $D$ is a constant. Note that a set of functions satisfying the above differential equation is given in Proposition 1 with $D=\frac{1}{2}$. However, it should be also noted that there are other triplets $(h, g, \xi)$ satisfying the conditions of Theorem 1 . 


\subsection{Characterization based on hazard function}

It is known that the hazard function, $h_{F}$, of a twice differentiable distribution function, $F$, satisfies the first order differential equation

$$
\frac{f^{\prime}(x)}{f(x)}=\frac{h_{F}^{\prime}(x)}{h_{F}(x)}-h_{F}(x) .
$$

For many univariate continuous distributions, this is the only characterization available in terms of the hazard function. The following characterization establish a non-trivial characterization of NGMW, in terms of the hazard function, which is not of the above trivial form.

proposition 2

Let $X: \Omega \rightarrow(0, \infty)$ be a continuous random variable. The pdf of $X$ is (6) if and only if its hazard function $h_{F}(x)$ satisfies the differential equation

$$
\begin{aligned}
& h_{F}^{\prime}(x)-\alpha\left(\beta+\gamma \lambda x^{\gamma-1}\right) h_{F}(x) \\
& =\alpha \mathrm{e}^{-\alpha \beta x-\alpha \lambda x^{\gamma}} \frac{d}{d x}\left\{\frac{\left(\beta+\gamma \lambda x^{\gamma-1}\right)}{\left(1-\mathrm{e}^{-\beta x-\lambda x^{\gamma}}\right)\left[\left(1-\mathrm{e}^{-\beta x-\lambda x^{\gamma}}\right)^{\alpha}+\theta \mathrm{e}^{-\alpha \beta x-\alpha \lambda x^{\gamma}}\right]}\right\}, x>0,
\end{aligned}
$$

with boundary condition $\lim _{x \rightarrow \infty} h_{F}(x)=0$.

Proof

If $X$ has pdf (6), then clearly the above differential equation holds. Now, if the differential equation holds, then

$$
\begin{aligned}
& \frac{d}{d x}\left\{\mathrm{e}^{-\alpha \beta x-\alpha \lambda x^{\gamma}} h_{F}(x)\right\} \\
& =\alpha \mathrm{e}^{-2\left(\alpha \beta x+\alpha \lambda x^{\gamma}\right)} \frac{d}{d x}\left\{\frac{\left(\beta+\gamma \lambda x^{\gamma-1}\right)}{\left(1-\mathrm{e}^{-\beta x-\lambda x^{\gamma}}\right)\left[\left(1-\mathrm{e}^{-\beta x-\lambda x^{\gamma}}\right)^{\alpha}+\theta \mathrm{e}^{-\alpha \beta x-\alpha \lambda x^{\gamma}}\right]}\right\},
\end{aligned}
$$

or

$$
h_{F}(x)=\frac{\alpha\left(\beta+\gamma \lambda x^{\gamma-1}\right) \mathrm{e}^{-\alpha \beta x-\alpha \lambda x^{\gamma}}}{\left(1-\mathrm{e}^{-\beta x-\lambda x^{\gamma}}\right)\left[\left(1-\mathrm{e}^{-\beta x-\lambda x^{\gamma}}\right)^{\alpha}+\theta \mathrm{e}^{-\alpha \beta x-\alpha \lambda x^{\gamma}}\right]},
$$

which is the hazard function of the NGMW distribution.

\subsection{Characterization in terms of the reverse hazard function}

The reverse hazard function, $r_{F}$, of a twice differentiable distribution function, $F$, is defined as

$$
r_{F}(x)=\frac{f(x)}{F(x)}, x \in \text { support of } F
$$

proposition 3

Let $X: \Omega \rightarrow(0, \infty)$ be a continuous random variable. The pdf of $X$ is (6) if and only if its reverse hazard function $r_{F}(x)$ satisfies the differential equation

$$
\begin{aligned}
& r_{F}^{\prime}(x)-\alpha\left(\beta+\gamma \lambda x^{\gamma-1}\right) r_{F}(x) \\
& =\alpha \theta \mathrm{e}^{-\alpha \beta x-\alpha \lambda x^{\gamma}} \frac{d}{d x}\left\{\frac{\left(\beta+\gamma \lambda x^{\gamma-1}\right)}{\left(1-\mathrm{e}^{-\beta x-\lambda x^{\gamma}}\right)\left[\left(1-\mathrm{e}^{-\beta x-\lambda x^{\gamma}}\right)^{\alpha}+\theta \mathrm{e}^{-\alpha \beta x-\alpha \lambda x^{\gamma}}\right]}\right\},
\end{aligned}
$$

with boundary condition $\lim _{x \rightarrow \infty} r_{F}(x)=0$. 
Proof

If $X$ has pdf (6), then clearly the above differential equation holds. Now, if this differential equation holds, then

$$
\begin{aligned}
& \frac{d}{d x}\left\{\mathrm{e}^{-\alpha \beta x-\alpha \lambda x^{\gamma}} r_{F}(x)\right\} \\
& =\alpha \theta \mathrm{e}^{-2\left(\alpha \beta x+\alpha \lambda x^{\gamma}\right)} \frac{d}{d x}\left\{\frac{\left(\beta+\gamma \lambda x^{\gamma-1}\right)}{\left(1-\mathrm{e}^{-\beta x-\lambda x^{\gamma}}\right)\left[\left(1-\mathrm{e}^{-\beta x-\lambda x^{\gamma}}\right)^{\alpha}+\theta \mathrm{e}^{-\alpha \beta x-\alpha \lambda x^{\gamma}}\right]}\right\},
\end{aligned}
$$

or

$$
r_{F}(x)=\frac{\alpha \theta\left(\beta+\gamma \lambda x^{\gamma-1}\right) \mathrm{e}^{-\alpha \beta x-\alpha \lambda x^{\gamma}}}{\left(1-\mathrm{e}^{-\beta x-\lambda x^{\gamma}}\right)\left[\left(1-\mathrm{e}^{-\beta x-\lambda x^{\gamma}}\right)^{\alpha}+\theta \mathrm{e}^{-\alpha \beta x-\alpha \lambda x^{\gamma}}\right]},
$$

which is the reverse hazard function of the OLLMW distribution.

\section{Estimation, inference and goodness-of-fit statistics}

Estimating the unknown parameters of a distribution is an essential issue in applied statistics. In this section, we obtain the maximum likelihood (ML) estimation of the parameters of the NGMW distribution based on a random sample $X_{1}, \ldots, X_{n}$ from this distribution. Further, five goodness-of-fit statistics are given to compare the density estimates and selection of the models.

\subsection{Maximum Likelihood Estimation}

By using the loglikelihood of the sample in conjunction with the NMaximize command in the symbolic computational package Mathematica, we can estimate the unknown parameters of a distribution. Given the observed values $x_{i}, i=1, \ldots, n$ of the taken sample from the NGMW distribution, the maximum likelihood estimators (MLEs) of the parameters are obtained by maximization of the log-likelihood function given by

$$
\begin{aligned}
\ell\left(x_{i} ; \Theta\right)= & \ell\left(x_{i}, \alpha, \beta, \theta, \lambda, \gamma\right)=n \log (\alpha \theta)+\sum_{i=1}^{n} \log \left(\beta+\gamma \lambda x_{i}^{\gamma-1}\right) \\
& +\alpha \sum_{i=1}^{n} \log \left(1-t_{i}\right)+(\alpha-1) \sum_{i=1}^{n} \log \left(t_{i}\right)-2 \sum_{i=1}^{n} \log \left(t_{i}^{\alpha}+\theta\left(1-t_{i}\right)^{\alpha}\right)
\end{aligned}
$$

where $t_{i}=1-\mathrm{e}^{-\beta \mathrm{x}-\lambda \mathrm{x}_{\mathrm{i}}^{\gamma}}$ and the associated nonlinear loglikehood system $\frac{\partial \ell(\Theta)}{\partial \theta}=0$, where

$$
\begin{aligned}
\frac{\partial \ell(\Theta)}{\partial \alpha} & =\frac{n}{\alpha}-\sum_{i=1}^{n} \log \left(t_{i}\left(1-t_{i}\right)\right)-2 \sum_{i=1}^{n} \frac{t_{i}^{\alpha} \log \left(t_{i}\right)+\theta\left(1-t_{i}\right)^{\alpha} \log \left(1-t_{i}\right)}{t_{i}^{\alpha}+\theta\left(1-t_{i}\right)^{\alpha}}=0 \\
\frac{\partial \ell(\Theta)}{\partial \beta} & =\sum_{i=1}^{n} \frac{1}{\beta+\gamma \lambda x_{i}^{\gamma}}-\alpha \sum_{i=1}^{n} \frac{t_{i}^{(\beta)}}{1-t_{i}}+\alpha \sum_{i=1}^{n} \frac{t_{i}^{(\beta)}}{t_{i}}-2 \alpha \sum_{i=1}^{n} t_{i}^{(\beta)} \frac{t_{i}^{\alpha-1}-\theta\left(1-t_{i}\right)^{\alpha-1}}{t_{i}^{\alpha}+\theta\left(1-t_{i}\right)^{\alpha}}=0, \\
\frac{\partial \ell(\Theta)}{\partial \lambda} & =\sum_{i=1}^{n} \frac{\gamma x_{i}^{\gamma-1}}{\beta+\gamma \lambda x_{i}^{\gamma}}-\alpha \sum_{i=1}^{n} \frac{t_{i}^{(\lambda)}}{1-t_{i}}+\alpha \sum_{i=1}^{n} \frac{t_{i}^{(\lambda)}}{t_{i}}-2 \alpha \sum_{i=1}^{n} t_{i}^{(\lambda)} \frac{t_{i}^{\alpha-1}-\theta\left(1-t_{i}\right)^{\alpha-1}}{t_{i}^{\lambda}+\theta\left(1-t_{i}\right)^{\alpha}}=0, \\
\frac{\partial \ell(\Theta)}{\partial \gamma} & =\lambda \sum_{i=1}^{n} \frac{x_{i}^{\gamma-1}\left[1+\gamma \log \left(x_{i}\right)\right]}{\beta+\gamma \lambda x_{i}^{\gamma}}-\alpha \sum_{i=1}^{n} \frac{t_{i}^{(\gamma)}}{1-t_{i}}+\alpha \sum_{i=1}^{n} \frac{t_{i}^{(\gamma)}}{t_{i}} \\
& -2 \alpha \sum_{i=1}^{n} t_{i}^{(\gamma)} \frac{t_{i}^{\alpha-1}-\theta\left(1-t_{i}\right)^{\alpha-1}}{t_{i}^{\alpha}+\theta\left(1-t_{i}\right)^{\alpha}}=0,
\end{aligned}
$$




$$
\frac{\partial \ell(\Theta)}{\partial \theta}=\frac{n}{\theta}-2 \sum_{i=1}^{n} \frac{\left(1-t_{i}\right)^{\alpha}}{t_{i}^{\lambda}+\theta\left(1-t_{i}\right)^{\alpha}}=0
$$

where

$$
\begin{aligned}
t_{i}^{(\lambda)} & =x_{i}^{\gamma}\left(1-t_{i}\right) \\
t_{i}^{(\beta)} & =x_{i}\left(1-t_{i}\right) \\
t_{i}^{(\gamma)} & =x_{i}^{\gamma}\left(1-t_{i}\right) \log \left(x_{i}\right)
\end{aligned}
$$

By solving the above equations simultaneously, we obtain the MLEs of the parameters. The numerical iterative techniques may be used for estimating the parameters and the global maxima of the log-likelihood is possible to investigate by putting different starting values for the parameters. The information matrix will be required for interval estimation. The elements of the $5 \times 5$ total observed information matrix $J(\Theta)=\operatorname{Jrs}(\Theta)$ for $r, s=$ $\alpha, \beta, \lambda, \gamma, \theta$, can be obtained from authors upon request. Under conditions that are fulfilled for parameters in the interior of the parameter space but not on the boundary, the asymptotic distribution of $\sqrt{n}(\hat{\Theta}-\Theta)$ is $N_{5}\left(O, I(\theta)^{-1}\right)$, where $I(\Theta)=E\{J(\Theta)\}$ is the expected information matrix. The approximate multivariate normal $N_{5}\left(O, I(\Theta)^{-1}\right)$ distribution, where $I(\Theta)^{-1}$ is the inverse observed information matrix evaluated at $\Theta=\hat{\Theta}$, can be used to set up the approximate confidence intervals of the distribution parameters.

\section{Simulation study}

The performance of the MLEs of the NGMW distribution with respect to sample size $\mathrm{n}$ is considered. Simulation study is done based on the following steps:

1. generate five thousand samples of size $\mathrm{n}$ from (6). This work is done by solving Equation (12) with numerical method based on R program ("uniroot" function).

2. compute the MLEs for the five thousand samples, say $\left(\hat{\alpha}_{i}, \hat{\beta}_{i}, \hat{\lambda}_{i}, \hat{\gamma}_{i}, \hat{\theta}_{i}\right)$ for $i=1,2, \ldots, 5000$.

3. compute the biases and mean squared errors given by

$$
\operatorname{Bias}_{\varepsilon}(n)=\frac{1}{5000} \sum_{i=1}^{5000}\left(\hat{\varepsilon}_{i}-\varepsilon\right)
$$

and

$$
M S E_{\varepsilon}(n)=\frac{1}{5000} \sum_{i=1}^{5000}\left(\hat{\varepsilon}_{i}-\varepsilon\right)^{2}
$$

for $\varepsilon=\alpha, \beta, \lambda, \gamma, \theta$.

We repeated these steps for $n=100,105, \ldots, 400$ with special case of parameters $\alpha=0.6, \beta=0.1, \lambda=0.1, \gamma=8$ and $\theta=2$. The shape of pdf for this case of parameters is bimodal and it is presented in Figure 1 . So computing $\operatorname{bias}_{\varepsilon}(n)$ and $M S E_{\varepsilon}(n)$ for $\varepsilon=\alpha, \beta, \lambda, \gamma, \theta$ and $n=100,105, \ldots, 400$.

Figure 3 shows how the five biases vary with respect to $n$. The biases for each parameter either decrease or increase to zero as $n \rightarrow \infty$.

Figure 4 shows how the five mean squared errors vary with respect to $n$. The mean squared errors for each parameter decrease to zero as $n \rightarrow \infty$.

The reported observations in both figures are for only one choice for $(\alpha, \beta, \lambda, \gamma, \theta)$, namely $(\alpha, \beta, \lambda, \gamma, \theta)=$ $(0.6,0.1,0.1,0.1,8,2)$. The results, however were similar for a wide range of other choices for $(\alpha, \beta, \lambda, \gamma, \theta)$. In particular, 1) the biases for each parameter either decreased or increased to zero and appeared reasonably small at $\mathrm{n} \geq 350$, 2) the mean squared errors for each parameter decreased to zero and appeared reasonably small at $\mathrm{n} \geq$ 350 . 

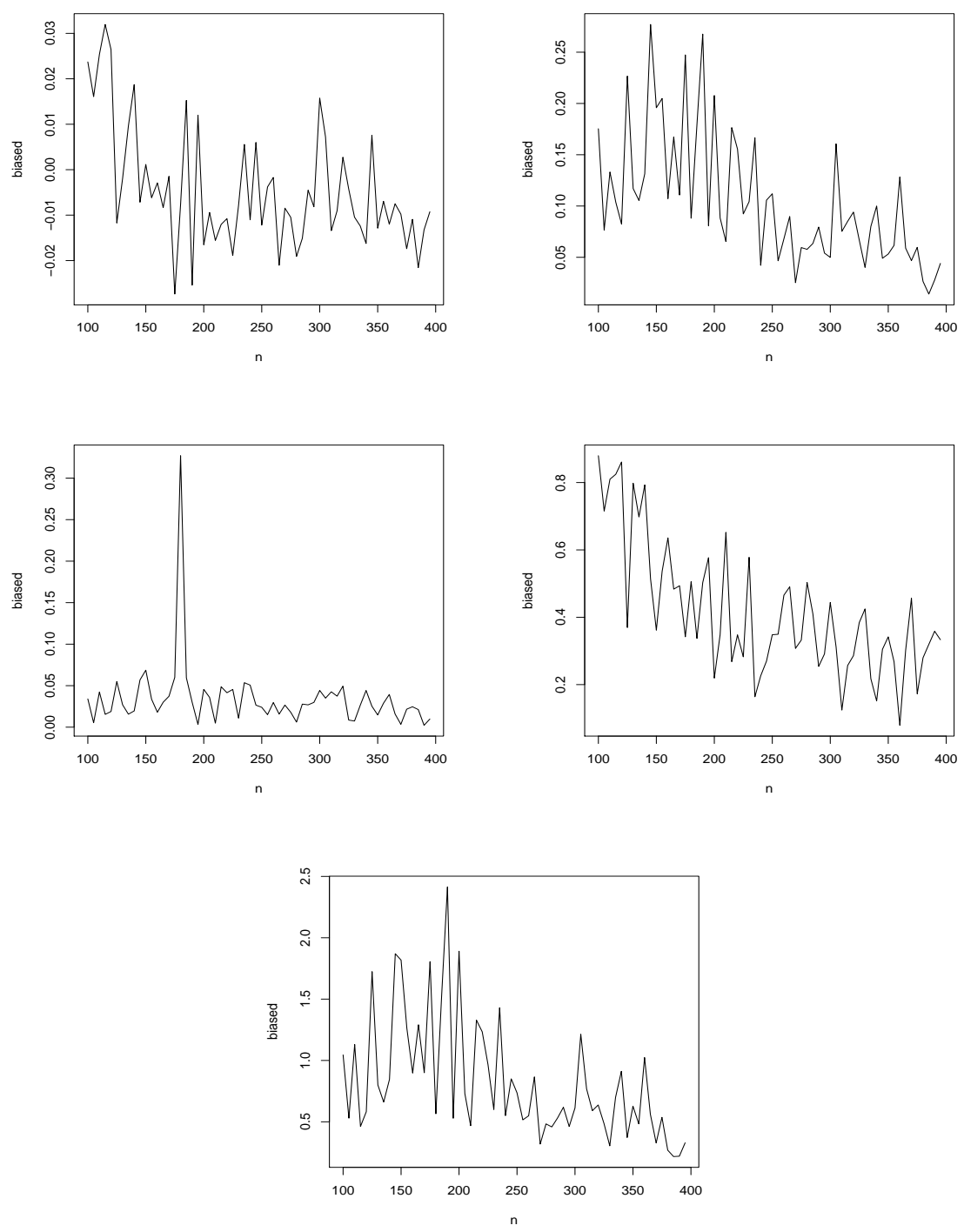

Figure 3. $\operatorname{bias}_{\alpha}(n)$ (top left), $\operatorname{bias}_{\beta}(n)$ (top right), $\operatorname{bias}_{\lambda}(n)$ (middle left), $\operatorname{bias}_{\gamma}(n)$ (middle right) and $\operatorname{bias}_{\theta}(n)$ (bottom left) versus $n=100,105, \ldots, 400$.

\section{Applications}

In this section, we compare the NGMW model with other related lifetime models, namely: generalized modified Weibull (GMW) [3], Mcdonald Lomax (McLomax) [14], beta modified Weibull (BMW) [18] and transmuted generalized modified Weibull (TGMW) [5] distributions. To do so, we make use of two real data sets: first, the carbon fibre data [4] and, secondly, the bladder cancer data [13]. More specifically, the fitted models are (for $x>0)$ :

- The GMW density function [3]

$$
f(x)=\varphi \alpha x^{\gamma-1}(\gamma+\lambda x) \mathrm{e}^{\lambda x-\alpha x^{\gamma} \mathrm{e}^{\lambda x}}\left(1-\mathrm{e}^{-\alpha x^{\gamma} \mathrm{e}^{\lambda x}}\right)^{\varphi-1},
$$



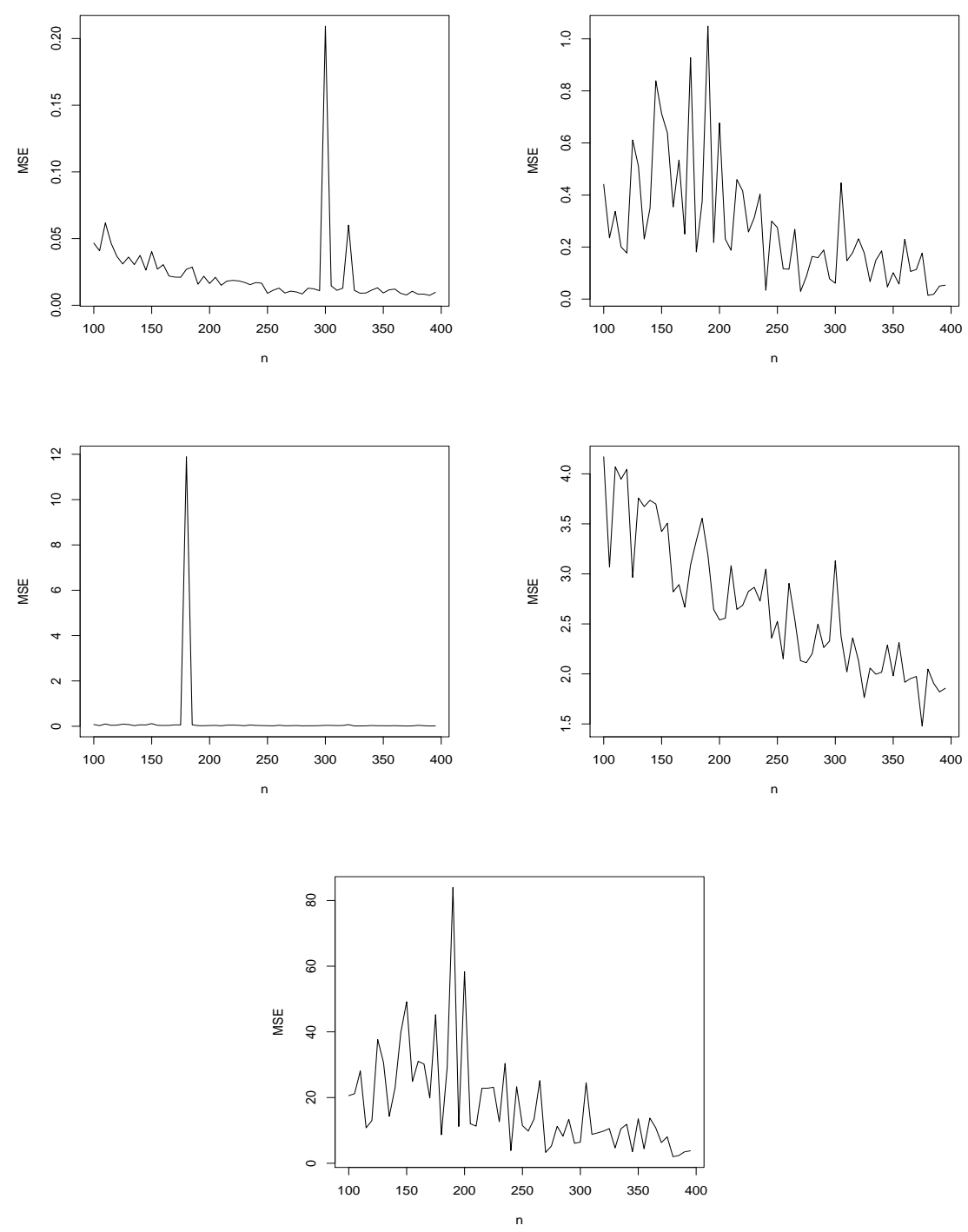

Figure 4. $M S E_{\alpha}(n)$ (top left), $M S E_{\beta}(n)$ (top right), $M S E_{\lambda}(n)$ (middle left), $M S E_{\gamma}(n)$ (middle right) and $M S E_{\theta}(n)$ (bottom left) versus $n=100,105, \ldots, 400$.

- The McLomax density function [14]

$$
f(x)=\frac{c \alpha \beta^{\alpha}(\beta+x)^{-(\alpha+1)}}{\operatorname{Beta}\left(a c^{-1}, b\right)}\left\{1-\left(\frac{\beta}{\beta+x}\right)^{\alpha}\right\}^{a-1}\left[1-\left\{1-\left(\frac{\beta}{\beta+x}\right)^{\alpha}\right\}^{c}\right]^{b-1},
$$

- The BMW density function [18]

$$
f(x)=\frac{\alpha x^{\gamma-1}(\gamma+\lambda x) \mathrm{e}^{\lambda x}\left(1-\mathrm{e}^{-\alpha x^{\gamma} \mathrm{e}^{\lambda x}}\right)^{a-1} \mathrm{e}^{-b \alpha x^{\gamma} \mathrm{e}^{\lambda x}}}{\operatorname{Beta}(a, b)},
$$


Table 2. MLEs (standard errors in parentheses) for the carbon fibres

\begin{tabular}{llllll}
\hline Distributions & Parameter & estimates & & & \\
\hline $\operatorname{GMW}(\varphi, \alpha, \gamma, \lambda)$ & 5.49894 & 0.43639 & 0.14811 & 0.51628 & \\
$\operatorname{McLomax}(\alpha, \beta, a, b, c)$ & $(8.02208)$ & $(0.64986)$ & $(0.53839)$ & $(0.16932)$ & \\
& $(16.155)$ & $(14.9998$ & 3.37645 & 1499.98 & 5.43418 \\
$\operatorname{BMW}(\alpha, \gamma, \lambda, a, b)$ & 0.44730 & 0.13899 & $0.79071)$ & $(7941.2)$ & $(3.49922)$ \\
& $(0.72868)$ & $(0.54698)$ & $(0.46150)$ & 5.87258 & 1.12967 \\
$\operatorname{TGMW}(\alpha, \varphi, \gamma, \lambda, \eta)$ & 0.19212 & 3.31948 & 0.27486 & $0.58561)$ & $(2.95269)$ \\
& $(0.40783)$ & $(4.54722)$ & $(0.82386)$ & $(0.23501)$ & 0.67440 \\
$\operatorname{NGMW}(\alpha, \beta, \lambda, \gamma, \theta)$ & 1.312813 & 0.739076 & 0.088051 & 2.211504 & 43.07359 \\
& $(1.28462)$ & $(2.74113)$ & $(0.942847)$ & $(4.20357)$ & $(207.414)$ \\
\hline
\end{tabular}

Table 3. Goodness-of-fit statistics for the carbon fibres

\begin{tabular}{lll}
\hline Distributions & $A^{*}$ & $W^{*}$ \\
\hline $\operatorname{GMW}(\varphi, \alpha, \gamma, \lambda)$ & 0.38543 & 0.06279 \\
$\operatorname{McLomax}(\alpha, \beta, a, b, c)$ & 0.49648 & 0.08398 \\
$\operatorname{BMW}(\alpha, \gamma, \lambda, a, b)$ & 0.38423 & 0.06261 \\
$\operatorname{TGMW}(\alpha, \varphi, \gamma, \lambda, \eta)$ & 0.33211 & 0.05279 \\
$\operatorname{NGMW}(\alpha, \beta, \lambda, \gamma, \theta)$ & $\mathbf{0 . 2 6 5 4 1}$ & $\mathbf{0 . 0 3 9 4 8}$ \\
\hline
\end{tabular}

- The TGMW density function [5]

$$
f(x)=\varphi \alpha x^{-1+\gamma}(\gamma+x \lambda) \mathrm{e}^{-\mathrm{e}^{x \lambda} x^{\gamma} \alpha+x \lambda}\left(1-\mathrm{e}^{-\mathrm{e}^{x \lambda} x^{\gamma}}\right)^{-1+\varphi}\left\{1+\eta-2\left(1-\mathrm{e}^{-\mathrm{e}^{x \lambda} x^{\gamma} \alpha}\right)^{\varphi} \eta\right\} .
$$

\subsection{The Carbon fibre data}

The first data set which is uncensored pertains to the breaking stress of carbon fibres (in Gba) as reported in [4].

\subsection{The bladder cancer data}

The second data set represents the remission times (in months) of a random sample of 128 bladder cancer patients as reported in Lee ${ }_{W}$ ang : 2003.

The estimated pdf's and cdf's of the TGMW model are plotted in Figures 4 and 5 for the carbon fibres and cancer data, respectively. The estimates of the parameters as well as the values of the Anderson-Darling $\left(A^{*}\right)$ and Cramér-von Mises $\left(W^{*}\right)$ statistics are listed in Tables 2 to 5. We note that the TGMW model provides the best fit for both data sets.

\section{Conclusions}

There has been a growing interest among statisticians and applied researchers in constructing flexible lifetime models in order to improve the modelling of survival data. As a result, significant progress has been made towards the generalization of the traditional Weibull model. In this paper, we propose a five-parameter model named the odd log-logistic Marshal-Olkin modified Weibull (NGMW), which is obtained by applying the odd loglogistic Marshal-Olkin technique to the modified Weibull model. The new model extends several important lifetime distributions. We studied some of its statistical properties and obtained representations of the positive, negative and factorial moments, as well as the quantile function and the density of the order statistics. The proposed distribution as applied to two actual data sets turned out to provide better fits than other competing lifetime models. 
Table 4. MLEs (standard errors in parentheses) for the bladder cancer data

\begin{tabular}{llllll}
\hline Distributions & Parameter & estimates & & & \\
\hline $\operatorname{GMW}(\varphi, \alpha, \gamma, \lambda)$ & 2.79601 & 0.45369 & 0.65441 & $5.8 \times 10^{-13}$ & \\
$\operatorname{McLomax}(\alpha, \beta, a, b, c)$ & $(1.85772)$ & $(0.37182)$ & $(0.24811)$ & $(0.00628)$ & \\
& $(3.364)$ & 11.2929 & 1.5060 & 4.1886 & 2.1046 \\
$\operatorname{BMW}(\alpha, \gamma, \lambda, a, b)$ & 0.46965 & 0.66613 & $(0.243)$ & $(25.029)$ & $(3.079)$ \\
& $(0.47875)$ & $(0.31225)$ & $(0.00639)$ & $(2.02018)$ & 0.90825 \\
$\operatorname{TGMW}(\alpha, \varphi, \gamma, \lambda, \eta)$ & 0.25215 & 2.24129 & 0.72431 & $3.4 \times 10^{-11}$ & $(1.52196)$ \\
& $(0.31749)$ & $(1.74023)$ & $(0.38549)$ & $(0.00795)$ & $(0.35566)$ \\
$\operatorname{NGMW}(\alpha, \beta, \lambda, \gamma, \theta)$ & 1.994473 & 0.015967 & 0.022496 & 0.417419 & 0.026061 \\
& $(1.22619)$ & $(0.033625)$ & $(0.051937)$ & $(0.338987)$ & $(0.123865)$ \\
\hline
\end{tabular}

Table 5. Goodness-of-fit statistics for the bladder cancer data

\begin{tabular}{lll}
\hline Distributions & $A_{0}^{*}$ & $W_{0}^{*}$ \\
\hline GMW $(\varphi, \alpha, \gamma, \lambda)$ & 0.27198 & 0.04050 \\
McLomax $(\alpha, \beta, a, b, c)$ & 1.81435 & 0.3550 \\
BMW $(\alpha, \gamma, \lambda, a, b)$ & 0.27197 & 0.04051 \\
TGMW $(\alpha, \varphi, \gamma, \lambda, \eta)$ & 0.18733 & 0.02732 \\
NGMW $(\alpha, \beta, \lambda, \gamma, \theta)$ & $\mathbf{0 . 0 8 7 5 3}$ & $\mathbf{0 . 0 1 3 6 4}$ \\
\hline
\end{tabular}
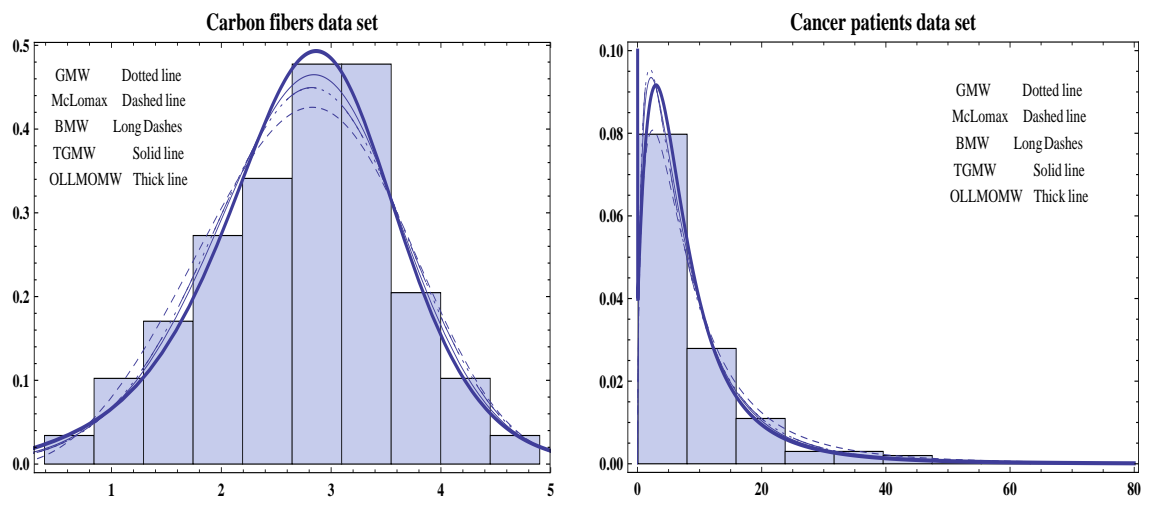

Figure 5. The estimated densities superimposed on the histogram

\section{Appendix A. Meijer $G$-function}

The symbol $G_{p, q}^{m, n}(\cdot \mid \cdot)$ denotes Meijer's $G$-function (MEIJ) which is defined in terms of a Mellin-Barnes integral as

$$
G_{p, q}^{m, n}\left(z \mid \begin{array}{c}
a_{1}, \cdots, a_{p} \\
b_{1}, \cdots, b_{q}
\end{array}\right)=\frac{1}{2 \pi \mathrm{i}} \oint_{\mathfrak{C}} \frac{\prod_{j=1}^{m} \Gamma\left(b_{j}-s\right) \prod_{j=1}^{n} \Gamma\left(1-a_{j}+s\right)}{\prod_{j=m+1}^{q} \Gamma\left(1-b_{j}+s\right) \prod_{j=n+1}^{p} \Gamma\left(a_{j}-s\right)} z^{s} \mathrm{ds},
$$

where $0 \leq m \leq q, 0 \leq n \leq p$ and the poles $a_{j}, b_{j}$ are such that no pole of $\Gamma\left(b_{j}-s\right), j=\overline{1, m}$ coincides with any pole of $\Gamma\left(1-a_{j}+s\right), j=\overline{1, n}$; i.e. $a_{k}-b_{j} \notin \mathbb{N}$, while $z \neq 0, \mathfrak{C}$ being a suitable integration contour, see [p. 143]Ref8 and MEIJ formoredetails. The $G$-function's Mathematica code reads

$$
\text { Meijerg }\left[\left\{\left\{a_{1}, \ldots, a_{n}\right\},\left\{a_{n+1}, \ldots, a_{p}\right\}\right\},\left\{\left\{b_{1}, \ldots, b_{m}\right\},\left\{b_{m+1}, \ldots, b_{q}\right\}\right\}, z\right] .
$$




\section{Appendix B.}

\section{Theorem 1}

Let $(\Omega, \mathcal{F}, \mathbf{P})$ be a given probability space and let $H=[d, e]$ be an interval for some $d<e$ $(d=-\infty, e=\infty$ might as well be allowed). Let $X: \Omega \rightarrow H$ be a continuous random variable with the distribution function $F$ and let $g$ and $h$ be two real functions defined on $H$ such that

$$
\mathbf{E}[g(X) \mid X \geq x]=\mathbf{E}[h(X) \mid X \geq x] \xi(x), \quad x \in H,
$$

is defined with some real function $\eta$. Assume that $g, h \in C^{1}(H), \xi \in C^{2}(H)$ and $F$ is twice continuously differentiable and strictly monotone function on the set $H$. Finally, assume that the equation $\xi h=g$ has no real solution in the interior of $H$. Then $F$ is uniquely determined by the functions $g, h$ and $\xi$, particularly

$$
F(x)=\int_{a}^{x} C\left|\frac{\xi^{\prime}(u)}{\xi(u) h(u)-g(u)}\right| \exp (-s(u)) \mathrm{d} u
$$

\section{REFERENCES}

1. Alizadeh, M., Nematollahi, A., Altun, E.,\& Rasekhi, M. , A Study on A New Type 1 Half-Logistic Family of Distributions and Its Applications, Statistics, Optimization \& Information Computing 8.4 ,pp934-949, 2020.

2. Bebbington, Mark, Chin-Diew Lai, and Riardas Zitikis., A flexible Weibull extension, Reliability Engineering \& System Safety 92.6 ,pp 719-726, 2007.

3. J.M.F. Carrasco, M.M. Edwin Ortega, G.M.Cordeiro, A generalized modified Weibull distribution for lifetime modelling, Computational Statistics and Data Analysis, 53 ,pp450-462, 2008.

4. G.M. Cordeiro, E.M.M. Ortega, A.J. Lemonte The exponential-Weibull lifetime distribution, Journal of Statistical Computation and Simulation, 84, pp 2592-2606, 2014.

5. G.M. Cordeiro, A. Saboor, M.N. Khan, S.B. Provost and E.M.M. Ortega The Transmuted Generalized Modified Weibull Distribution, Filomat 31.5, pp1395-1412, 2017.

6. H.A. David, H.N. Nagaraja Order statistics, John Wiley \& Sons, Inc. 1970

7. Glänzel, W. A characterization theorem based on truncated moments and its application to some distribution families. In Mathematical statistics and probability theory, . (pp. 75-84). Springer Netherlands, 1987.

8. Glänzel, W. Some consequences of a characterization theorem based on truncated moments, Statistics, 21(4), pp613-618, 1990.

9. J.U. Gleaton, J.D. Lynch Properties of generalized log-logistic families of lifetime distributions, Journal of Probability and Statistical Science 4, no. 1,pp 51-64, 2006.

10. Gleaton, James U., and James D. Lynch Extended Generalized Log-logistic Families of Lifetime Distributions with an Application, J. Probab. Stat. Sci 8.1 ,pp 1-17, 2010.

11. M.N. Khan, The modified beta Weibull distribution, Hacettepe Journal of Mathematics and Statistics, 44 ,pp 1553-1568, 2015.

12. Benkhelifa, The Weibull Birnbaum-Saunders distribution and its applications, Statistics, Optimization \& Information Computing, 2020.

13. Lee E, Wang J. Statistical Methods for Survival Data Analysis, Wiley \& Sons: New York; 2003.

14. Lemonte AJ, Cordeiro GM. An extended Lomax distribution, Statistics: A Journal of Theoretical and Applied Statistics. 47, pp 800-816, 2013.

15. Y.L. Luke, The Special Functions and Their Approximations, San Diego: Academic Press, 1969.

16. C.S. Meijer On the G-function I-VIII, Proc. Kon. Ned. Akad. Wet, 49 227-237, 344-356, 457-469, 632-641, 765-772, 936-943, 1063-1072, 1165-1175, 1946.

17. G. S. Mudholkar, D. K. Srivastava, M. Friemer The exponentiated Weibull family: A reanalysis of the bus-motor-failure data, Technometrics, 37, 436-445, 1995.

18. Silva GO, Edwin MM Ortega and Cordeiro GM. The beta modified Weibull distribution, Lifetime. Data. Anal. 16, 409-430, 2010.

19. A. Saboor, S.B. Provost, M. Ahmad, The moment generating function of a bivariate gamma-type distribution,, Applied Mathematics and Computation, 218(24), 11911-11921, 2012.

20. M. Xie, C.D. Lai, Reliability analysis using an additive Weibull model with bathtub-shaped failure rate function, Reliability Engineering and System Safety, 52 ,87-93, 1995.

21. W.H. Von Alven, Reliability engineering, Prentice Hall, 1964.

22. G.R. Aryal, C.P. Tsokos, Transmuted Weibull Distribution: A Generalization of the Weibull Probability Distribution, EUROPEAN JOURNAL OF PURE AND APPLIED MATHEMATICS, 4 , 89-102, 2011.

23. V. Choulakian, M.A. Stephens, Goodness-of-fit for the generalized Pareto distribution, Technometrics, 43, 478-C484, 2001

24. M.S. Khan, R. King, Transmuted Modified Weibull Distribution: A Generalization of the Modified Weibull Probability Distribution, EUROPEAN JOURNAL OF PURE AND APPLIED MATHEMATICS, 6, 66-88, 2013

25. Pogány, T. K., Saboor, A. and Provost, S. The Marshall-Olkin exponential Weibull distribution, Hacettepe Journal of Mathematics and Statistics, $44,1579-1594,2015$. 
26. Saboor, A., Alizadeh, M., Nauman Khan, M. and Ghosh, I. The Odd Log-Logistic Modified Weibull distribution, Mediterranean Journal of Mathematics 14.2 : 96, 2017.

27. S. Pundir, S. Arora, K. Jain, Bonferroni curve and the related statistical inference, Statistics and Probability Letters, 75 ,p140-C150, 2005.

28. A.M. Sarhan, M. Zain-din, Modified Weibull distribution, Applied Sciences, 11 , 123-136, 2009. 